Denis Dennehy ${ }^{\mathrm{a}}$, Laleh Kasaraian ${ }^{\mathrm{b}}$, Paidi O'Raghallaigh ${ }^{\mathrm{c}}$, Kieran Conboy ${ }^{\mathrm{a}}$, Dave Sammon ${ }^{\mathrm{c}}$, Patrick Lynch ${ }^{\mathrm{d}}$

a. Lero | The Irish Software Research Centre, National University of Ireland Galway, Galway, Ireland.

b. The School of Computer Science and Informatics, De Montfort University, Leicester, UK.

c. Cork University Business School, University College Cork, Cork, Ireland.

d. Texuna Technologies Ltd., London, UK.

\title{
A Lean Start-up Approach for Developing Minimum Viable Products in an Established Company
}

\begin{abstract}
The minimum viable product (MVP) is a fundamental concept of the Lean Start-up approach as it enables a company to quickly start the learning process by integrating feedback from early adopters. Although the MVP concept has evolved over the years, its application is most often reported in a start-up context, even though established companies struggle to develop MVPs. This study reports on the experience and lessons learned at Texuna Technologies, an established company, where the software product innovation team created a process map for developing MVPs. This is the first study that allows the original MVP approach to be extended and applied to established organisations.
\end{abstract}

Keywords: lean start-up, minimum viable product, product market fit

\section{INTRODUCTION}

The Lean Start-up approach has been promoted as a way to nurture product innovation in established companies (1). The Lean Start-up approach has received some attention from established companies including 3M, Telefonica, and General Electric. However, adapting an approach intended for a start-up context so that it can work effectively in established companies with established operating policies and procedures is challenging (2). For example, the development of a MVP, a fundamental concept of the Lean Start-up approach, which enables companies to quickly start the learning process by integrating feedback from early adopters (3) has been identified as a key challenge for established companies.

Reis (4) advocates that a company should commence building as quickly as possible with a MVP, which is a "version of the product that enables a full turn of the Build-Measure-Learn loop with the minimum amount of effort and least amount of development time" (p.77). When a company makes changes to the MVP, based on what they have learned about customers it is known as pivoting (5). It is through this iterative process of learning and pivoting that validation of a 'product-market fit' (PMF) is achieved. A PMF requires three criteria to be satisfied, (i) the customer is willing to pay for the product, (ii) there is an economically viable way to acquire customers, and (iii) the market is large enough for the business (6). Brown (7) advocates that a product must therefore fulfil and balance three overlapping criteria (i) desirability (what people want), (ii) feasibility (what is functionally possible), and (iii) viability (what is likely to become a sustainable business model). A business model describes the viability of a company in terms of how it fulfills its purpose, its goals and its ongoing plans for achieving them.

From a practice perspective, the overlapping criteria require a multidisciplinary approach that involves a diverse team of people with different skill-sets in order to provide a holistic solution (8). This study presents a process map for developing MVPs that achieve these three criteria (desirability, feasibility, and viability). 


\section{RELATED WORK ON THE LEAN START-UP APPROACH IN ESTABLISHED COMPANIES}

The Lean Start-up approach has been proposed as a new approach to innovation in situations where there is extreme uncertainty regarding who the customer is and what the customer's needs are. A start-up is defined (1) as "a human institution designed to create a new product or service under extreme uncertainty. That means entrepreneurs are everywhere and the Lean Start-up approach can work in any size company, even a very large company, in any sector or industry." A study of 170 business executives by (2) reports that $82 \%$ of them are using some elements of the Lean Start-up approach in their company and that developing a MVP has been a specific challenge.

The Lean Start-up approach has been shown to be quite effective (3) and is rapidly becoming the primary model for start-up innovation, overcoming challenges typically faced by startups, such as time and cost constraints, limited to a very small number or even a single entrepreneur, and facing competition from established organisations with diverse and multi skilled workforces, all of whom have access to a massive and diverse set of resources. However, despite this popular opinion, many established companies often face similar constraints.

The MVP is a fundamental concept of the Lean Start-up approach as it enables companies to quickly start the learning process by integrating feedback from early adopters. A review of literature by (4) identified twentytwo definitions of MVP in studies between 2001 and 2015. Whether or not the Lean Startup approach might support software product innovation in established companies and how best to approach it remains under studied $(5,6)$.

Previous studies that focused on the application of the Lean Start-up approach in established companies created frameworks that support the setting up of 'internal' startups. While such studies and associated frameworks provide much needed guidance on setting up internal lean start-ups, they do not support practitioners to develop MVPs.
While this study builds on earlier work by (7), it differs because the authors include the CEO of Texuna and the former Director of Research and Innovation (DRI) who provide reflections and lessons learned from their experience. To the best of our knowledge, it is the first study to empirically investigate the development of MVPs, using the Lean Start-up approach to developing MVPs in an established software company.

\section{References}

1. Ries, E. (2011) The lean startup: How today's entrepreneurs use continuous innovation to create radically successful businesses. Crown Books.

2. Kirsner, S. (2016) The barriers big companies face when they try to act like lean startups.

https://hbr.org/2016/08/the-barriersbig-companies-face-when-they-try-toact-like-lean-startups

3. Blank, S. (2013) Why the lean start-up changes everything. Harvard Business Review, 91(5), pp.63-72.

4. Lenarduzzi, V. and Taibi D. (2016) MVP explained: A systematic mapping study on the definitions of minimal viable product. In Proceedings of the 42th Euromicro Conference on Software Engineering and Advanced Applications (SEAA), Cyprus, Greece, 2016 (pp. 112-119). IEEE.

5. Edison, H., Smørsgård, N.M., Wang, X. and Abrahamsson, P. (2018) Lean Internal Startups for Software Product Innovation in Large Companies: Enablers and Inhibitors. Journal of Systems and Software, 135, pp.69-87.

6. Märijärvi, J., Hokkanen, L., Komssi, M., Kiljander, H., Xu, Y., Raatikainen, M., Seppänen, P., Heininen, J., KoivulahtiOjala, M., Helenius, M. and Järvinen, J., (2016) The cookbook for successful internal startups. DIGILE and N4S.

7. Dennehy. D., Kasraian, L., O' Raghallaigh, P. \& Conboy, K. (2016). Product Market Fit Frameworks for Lean Product Development. In R\&D Management Conference 2016 "From Science to Society: Innovation and Value Creation" 3-6 July 2016, Cambridge, UK. 


\section{BACKGROUND To TEXUNA}

Founded in London in 2000, Texuna has offices located in the UK, Ireland and Russia. Texuna has a strong reputation for designing scalable and secure data platforms that adhere to international standards (e.g. ISO 27001 for Information Security, ISO9001 for Quality Management, and ISO2000 for IT service management). Its client base includes both public and private sector clients from a range of industries and it supports in excess of 70,000 end-users dispersed across a large geographical area. In 2012 the company collaborated with University College Cork (Ireland) in establishing an Edtech Research Centre (ERC) for the purpose of investigating opportunities for future products and services using customer-centric design methods. The ERC was co-located with the company's Shared Services Centre. The CEO sought to explore new approaches that would enable the company to be more effective in bringing product ideas forward to successful product launches. The research team recommended the Lean Start-up approach to support the development of MVPs as the existing software development method (Scrum) was not supporting the team to develop multiple MVPs concurrently.

In its first year of operation the ERC adopted a Lean Start-up approach to successfully develop five MVPs. An example of one MVP was a student evaluation platform to administer high stake formative and summative examinations to students.

The university researchers, including two authors of this paper, were given full access to the ERC and were embedded in the day-to-day activities of the innovation team for a period of six months. During this time the frameworks emerged in tandem with the adaptation of the Lean Start-up approach within the ERC. For instance, the student evaluation platform was released as a MVP to a number of pilot sites (e.g. a private education entity, a local university, a training unit in a global pharmaceutical company). In addition, focus groups were held students, lecturers, administration and management staff, as well as those participating in the pilots. During the focus groups, participants interacted with the MVP and were presented with a number of questions. After the focus groups the pilot sites continued to use the MVPs and they provided daily feedback. Online usability testing of the MVP was also organised. The insights from these activities informed the evolution of the framework and process map presented in this paper.

\section{THE FRAMEWORKS EXPLAINED}

This section presents two frameworks that can assist established companies to develop MVPs. Figure 1 represents the three overlapping criteria (desirability, feasibility, viability) for a product to be successful and the iterative process between MVP and PMF. 


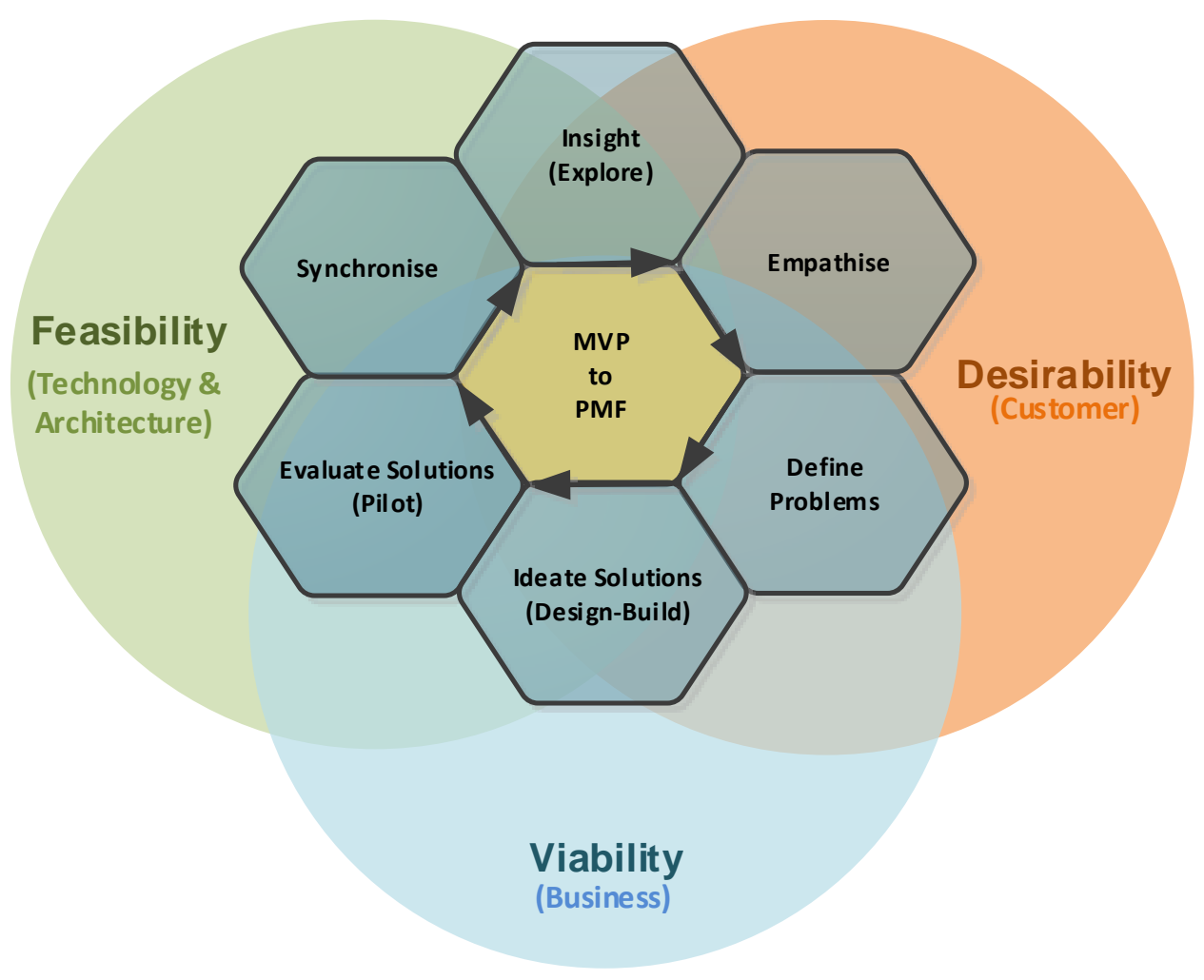

Figure 1: A MVP to PMF Conceptual Framework

The 'desirability' space develops the necessary collaboration with the stakeholders by asking "What do people desire?" and ultimately "How do people decide on new products?" A company needs to start by determining what would deliver value to the stakeholders. The 'viability' space clarifies the answer to the question "What is a sustainable business model?" The 'feasibility' space considers "What is technically and organisationally feasible?" The solutions that emerge at the end of this iterative process should be positioned at the intersection of these three dimensions (desirability, feasibility, and viability). It is here that sustainable innovation occurs as it is based on understanding and fulfilling the needs of the customer, creating products that are technically feasible, and sufficient value is generated for the company.

Building on Figure 1 and the experience at Texuna, a MVP process map (Figure 2) was developed. The MVP process map advocates a process that commences with gaining insights from current or potential customers; this is the primary motivation for creating a new product and forms the basis for developing the initial MVP. If the level of desirability is insufficient, then the company must decide to either pivot the problem (by moving to an alternative problem) or to end the exploratory phase completely. In most instances though, the desirability phase reveals various problems that were not envisaged by the design team during the ideation phase. Adding new features to the product, removing features not desired, or modifying features that were problematic for users subsequently refine the MVP. This results in a new version of the MVP and a second iteration of the evaluation process is required. This iterative evaluation process is repeated until the MVP moves closer to the PMF. Once the PMF is achieved, then evaluation is complete and a product launch is planned. 


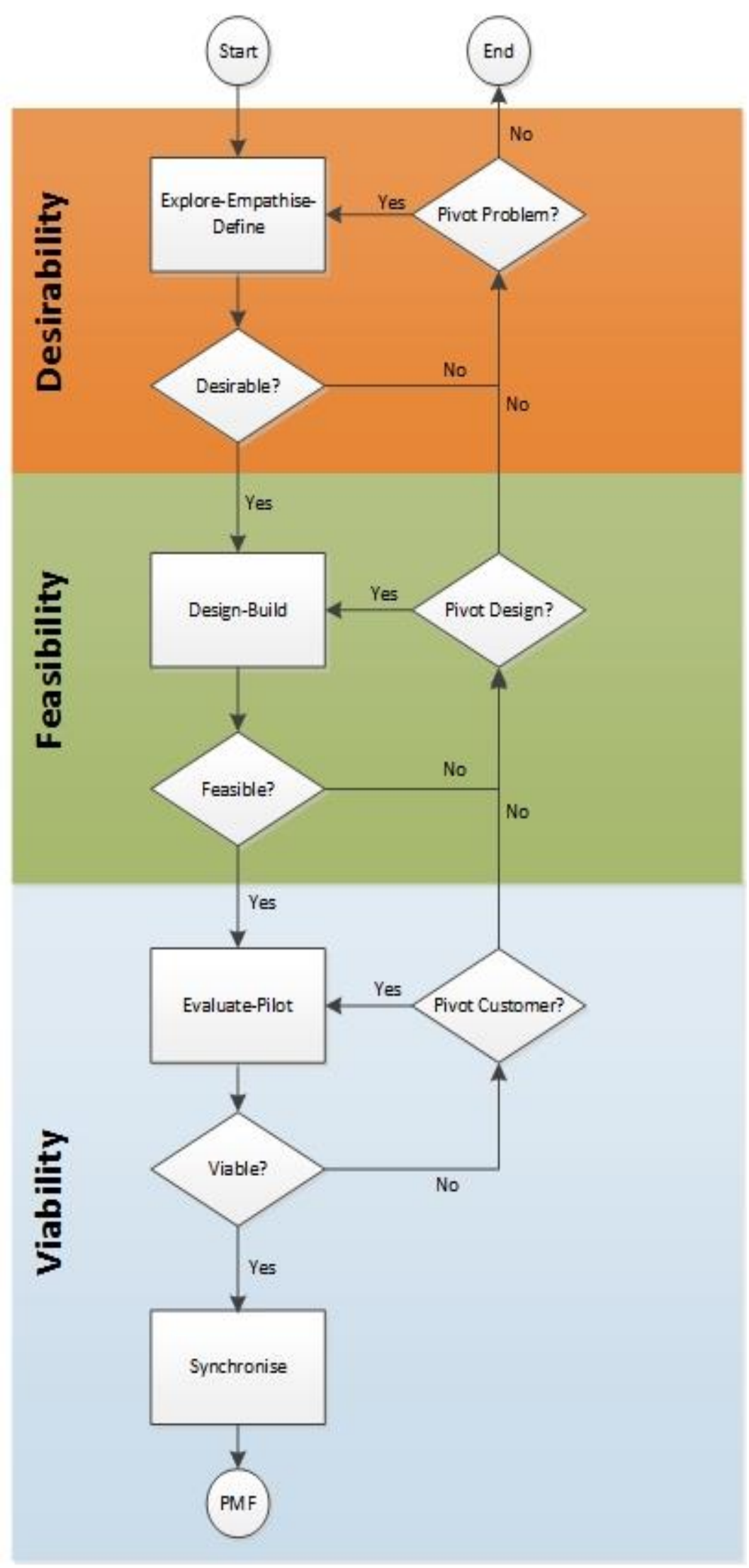

Figure 2: MVP Process Map 


\section{LESSONS LEARNED FROM ADOPTING THE MVP CONCEPT}

The natural inclination of the Texuna Edtech Research Centre (ERC) team (and especially those from a technical background) was to focus on questions of feasibility. They needed to be redirected to questions of desirability and viability. The Director of Research and Innovation (DRI) realised that the most likely failure points in the MVPs would be issues of 'desirability' and 'viability'. For this reason, three circles of equal size representing desirability, feasibility and viability were added to the MVP-PMF conceptual framework. In order to highlight the importance of developing the MVP to address the specific need of customers, the 'Empathise Step' was added. This placed a clear focus on taking the time to understand the needs of the customer and the context in which they would engage with the product.

The 'Evaluate-Pilot Step' initially included the word 'test' (Pilot Test Step). However, it was observed that the word 'test' gave the impression that the value of the MVP could be objectively judged in absolute terms through traditional software testing, whereas the opposite was found to be the situation. The concept of pilot sites became fundamental to the approach and to building empathy. In some situations a MVP that worked well did not necessarily meet the needs of a pilot site. In addition, an MVP that worked well in one pilot site did not necessarily work as well in another. The word 'evaluate' was found to better reflect the activity required to judge the relative value of the MVP.

Another interesting lesson was the natural instinct among some members of the innovation team to delay the release of the MVP to pilot sites. There was a strong culture within Texuna for delivering quality software and team members were often heard to judge themselves by the quality of their outputs. Employees were therefore keen to perfect those outputs before handing them over to pilot sites. In addition, MVPs were deemed by team members to have been a 'failure' when they were less well received by pilot sites even though many insightful lessons may have being learned from their release. The natural instinct was, therefore, for the MVPs to be seen as outputs rather than a means for learning - the focus thereby gradually creeping away from learning towards quality.

The early release of MVPs contributed, at times, to low morale within the team. The teams felt they were releasing low quality outputs that had not been fully developed or fully tested. The DRI had to consistently challenge this mind-set and in some cases he decided to release MVPs to pilot sites (even when he himself had some concerns about their quality) as he wished "to send out a message to his own team of the need for early customer involvement".

The addition of the 'Synchronise Step' was critical to the success of the MVP Process Map at Texuna. The innovation team successfully developed and evaluated five MVPs that provided large market opportunities for Texuna. However, a great deal of effort was needed in order to build the core capabilities required within Texuna to leverage these opportunities. For example, Texuna did not always have the sales and marketing bandwidth or skillsets to actively market a number of these MVPs, which differed from the traditional offerings of the company. The Synchronise Step was included to recognise the need for changes in the parent organisation in order to take advantage of the opportunities offered by the Lean Start-up approach. It could not be taken for granted that an established company can benefit from the Lean Start-up approach without internal changes (e.g. change in organisation culture, behaviours, systems, etc.).

It was noted that fatigue had set in within the innovation team whose members were often working on daily release cycles in response to faster feedback from a growing number of pilot sites. The fact the team was colocated with other company staff working at a slower pace created tension. Members of the innovation team felt they were being asked to shift from a 'large team mentality' with its clearly defined functional roles to a more 'flexible mentality' where they needed to 'roll up their sleeves' and take on different roles in order to get the job done. This suited some people but not all. For example, a senior developer saw this as eroding his 
'market value', which he felt, was based on producing high quality code using leading edge software tools. He wanted to be given clearly defined requirements rather than working in an environment where the needs of the customers emerged in an agile participatory way.

Team members were encouraged to make fast and courageous decisions. The cultural differences between the high-speed learning-based approach advocated by the Lean Start-up approach openly clashed with the slower paced culture at Texuna. It was increasingly apparent to the DRI that the former was not part of the dominant culture of the company and that the only way it would survive is with the continued support of the organisational leadership. Some internal stakeholders, however, advocated that the team should slow down and indeed others felt that it should be disbanded. As a compromise, the executive leadership requested that the speed be reduced and that other areas of the company would use some of the expertise within the team. For example, team members were increasingly asked to support the sales team in selling current products.

Throughout the project the researchers compiled a set of generic evaluation questions (see Table 1) that were collected from the discourses that took place at the ERC regarding the resulting MVPs. These questions being used to test the concepts of desirability, feasibility, and viability of a MVP.

Table 1: Evaluation questions

\begin{tabular}{|l|l|}
\hline \multirow{5}{*}{$\begin{array}{l}\text { Desirability } \\
\text { Lens }\end{array}$} & What is the participant's first impression of the MVP? \\
What are the perceived strengths and weaknesses of the MVP? \\
What benefits would the MVP provide to the participant? \\
What participant's pain point or desire does the MVP address? \\
How does the MVP compare to alternative options? \\
What level of proficiency is required to use the MVP? \\
Would the MVP fit in with the life of the participant? \\
$\begin{array}{ll}\text { Are there any obvious impediments to using the MVP? } \\
\text { Would the participant be willing to pay for the MVP? }\end{array}$ \\
\hline $\begin{array}{l}\text { Are we better placed than others to succeed with the MVP? } \\
\text { What would be involved in building the MVP? } \\
\text { What would be involved in entering the market? } \\
\text { What would be involved in scaling market share? } \\
\text { Can we protect the intellectual property in the MVP? } \\
\text { Do we have the required technical and market capabilities? } \\
\text { Do we have the required team to succeed? } \\
\text { Do we have the required partnerships to succeed? } \\
\text { Do we have sufficient team engagement? } \\
\text { Do we have sufficient leadership commitment? }\end{array}$ \\
$\begin{array}{l}\text { Does the MVP align with the organisation vision, values and goals? } \\
\text { Is there a viable business model for the MVP? } \\
\text { Is there sufficient revenue potential to justify the MVP? } \\
\text { Who will be willing to pay for the MVP and at what price point? } \\
\text { Is the market for the MVP flat, declining or growing? } \\
\text { Is there an adequate return on the investment? } \\
\text { How long before we break even with the MVP? } \\
\text { Can market share be grown and protected? } \\
\text { Is there a threat of new entrants and substitutes? } \\
\text { Who are the competitors and how powerful are they? } \\
\text { Is there sufficient finance available to succeed? }\end{array}$ \\
\hline
\end{tabular}


This set of evaluation questions supports both innovation teams and managers to balance discussions when evaluating their MVPs as it ensures that innovation teams do not have a 'rose tinted' view of their MVP, which may be technologically feasible but lack desirability and/or viability. Analyses of the answers to these questions can include quantitative techniques such as sentiment analysis and qualitative techniques such as thematic analysis.

\section{RECOMMENDATIONS}

We have observed how the MVP process map has strengthened the company's mental models of how to go about introducing new opportunities for learning from a MVP, although the capability to do so is perhaps building up at a slower pace. It is fair to say that some software product development team members have a greater appetite and aptitude for the Lean Start-up approach. We expect that this Lean Start-up oriented mindset will have a long-term effect as the frameworks have reshaped Texuna's current design practices and design philosophies.

The recommendations presented below are based on observations by the authors derived from the case studied but which are likely to be relevant to the management teams of other established companies interested in implementing a Lean Start-up approach.

Shared understanding about the purpose of a MVP: The MVP concept presented challenges for management and development teams as its purpose was misunderstood (e.g. MVP seen as a product to be delivered as opposed to a means for learning). This led to team members experiencing a sense of 'failure' when an MVP required pivots or a fear that colleagues or management would consider their outputs to be of low quality. Management and development teams need to be informed from the outset about the purpose of the MVP.

Balancing feasibility with desirability and viability: Members of the innovation team possessed strong technical skills, which enabled them to create software that showcased their technical know-how. However, efforts had to be made on a continuous basis to ensure that issues of desirability and viability were also being considered in the design of the MVPs.

Achieving PMF requires organisation-wide support: The Edtech Research Centre produced five MVPs, but not all of them ended up being released to the market. As Texuna did not have the sales and marketing capabilities to actively market these products, opportunities were lost. These capabilities need to be developed within the company in order to leverage emergent opportunities.

Changing organisational culture: The cultural differences between the high-speed learning approach clashed with the slower paced culture of Texuna. The agility of the innovation team and its ability to make prompt decisions challenged the status quo, which created tensions. Changing the organisational culture requires continued support of the organisational leadership and in certain instances a compromise may be required to satisfy all stakeholders.

There is a strong rationale underpinning each component of the MVP-PMF conceptual framework and the MVP process map. We would therefore, strongly discourage practitioners from adopting all components without reflection and tailoring. Instead, companies should ensure that the Lean Start-up approach is considered within its wider innovation management portfolio. Each component of the diagrams presented in this paper may be more relevant and critical in certain product/software design contexts than others. We encourage practitioners to reflect on each and to adopt or tailor as necessary in order to determine whether the Lean Start-up itself is suitable in that instance. 


\section{REFERENCES}

1. Märijärvi, J., Hokkanen, L., Komssi, M., Kiljander, H., Xu, Y., Raatikainen, M., Seppänen, P., Heininen, J., Koivulahti-Ojala, M., Helenius, M., and Järvinen, J. (2016) The cookbook for successful internal startups. DIGILE and N4S.

2. Kirsner, S. (2016) The barriers big companies face when they try to act like lean startups. https://hbr.org/2016/08/the-barriers-big-companies-face-when-they-try-to-act-like-leanstartups

3. Blank, S. (2007) The Four Steps to the Epiphany - Successful Startup Strategies for Products that Win. . https://web.stanford.edu/group/e145/cgibin/winter/drupal/upload/handouts/Four Steps.pdf.

4. Ries, E. (2011) The lean startup: How today's entrepreneurs use continuous innovation to create radically successful businesses. Crown Books.

5. Bosch, J., Olsson, HH., Björk, J., and Ljungblad, J. (2013) The early stage software startup development model: a framework for operationalizing lean principles in software startups. Lean Enterprise Software and Systems. Springer, pp. 1-15.

6. Cooper, B., and Vlaskovits P. (2010) The entrepreneur's guide to customer development: a "cheat sheet" to The four steps to the epiphany.[S1]: B. Cooper and P. Vlaskovitz.

7. Brown, T. (2009) Change by design: how design thinking transforms organizations and inspires innovation. New York: Harper Business.

8. Zupan, B., and Nabergoj, AS. (2012) Developing Design Thinking Skills in Entrepreneurship Education. . Leading Innovation Through Design, p. 555. Proceedings of the 2012 International Design Management Research Conference, Boston, USA. 